\title{
AVALIAÇÃO DO APROVEITAMENTO ESCOLAR DE PRE-ESCOLARES DO PROGRAMA CENTRO DE EDUCAÇÃO E ALIMENTAÇÃO DO PRÉ-ESCOLAR *
}

\author{
Maria José Pontieri ** \\ Maria Lucia Ferrari Cavalcanti $* * *$ \\ Yaro R. Gandra***
}

\begin{abstract}
PONTIERI, M. J. et al Avaliação do aproveitamento escolar de pré-escolares do Programa Centro de Educação e Alimentação do Pré-Escolar. Rev. Saúde públ., S. Paulo, 15(supl.): 148-58, 1981.

RESUMO: Foi avaliado o rendimento escolar dos pré-escolares que frequuentaram o Centro de Educação e Alimentação do Pré-Escolar (CEAPE) em comparação com um grupo controle, da mesma comunidade, mas que não freqüentou o programa. Ficou evidenciado que o aproveitamento escolar nas $1^{\text {s }} \mathrm{S}$ e $2^{\text {a }} \mathrm{S}$ séries do $1^{\circ}$ grau foi significantemente maior entre os pré-escolares "Ceapenses" que nos grupos controle "Não-Ceapenses". Portanto, além de cliferenças no desenvolvimento sócio-psicomotor mostradas em trabalhos anteriores, o programa CEAPE, apesar de simples e econômico, garante, também, melhor aproveitamento escolar aos que o frequientaram.
\end{abstract}

UNITERMOS: Pré-escolares, avaliação. Criança, desenvolvimento. Aprendizagem. CEAPE.

\section{INTRODUÇAOO}

No Brasil, como nas outras áreas em desenvolvimento, a criança em idade pré-escolar vem preocupando as autoridades de Saúde, Educação e Serviço Social, por razões justificadas ante um amplo conjunto de evidências.

Investigações realizadas em diversas partes do mundo evidenciam que a desnutrição protéico-calórica precoce e grave traz como consequiência direta um retardo no crescimento físico ${ }^{3,6}$ e no desenvolvimento mental 4,9,11,1:2 limitando, assim, a realização do potencial genético do ser humano e interferindo na capacidade de aprendizagem ${ }^{11}$.
Influências positivas do meio ambiente que circunda a criança ensejam-lhe oportunidade de enriquecimento, oferecendo-lhe diferentes tipos de estímulos. Chase e Martin 5 , referem que o efeito do meio familiar sobre - desenvolvimento intelectual da criança sugere que a inteligência da mãe afeta o sistema de linguagem que ela emprega em sua interação com os filhos; isto pode constituir fator critico no desenvolvimento da linguagem $e$ de habilidades cognitivas de pré-escolares.

A falta de atendimento integral, prévio e precoce a pré-escolares interfere negativa-

* Convênio 10/77 - INAN/DN/FSP/USP.

** Do Programa CEAPE - Departamento de Nutrição da Faculdade de Saúde Pública da USP - Av, Dr. Arnaldo, 715 - 01255 - São Paulo, SP - Brasil.

*** Do Departamento de Nutrição da Faculdade de Saúde Pública da USP - Av. Dr. Arnaldo, 715 - 01255 - Săo Paulo, SP - Brasil. 
PONTIERI, M.J. et al. Avaliação do aproveitamento escolar de pré-escolares do Programa Centro de Educação e Alimentação do Pré-Escolar. Rev. Saúde públ., S. Paulo, 15(supl.): 148-58,1981.

mente no processo de maturação e na prontidão da criança, prejudicando sua capacidade funcional e de aprendizagem. Crianças que não receberam atenção suficiente $e$ adequada na idade que antecede seu ingresso $\dot{a}$ escola, ai chegam sem as habilidades indispensáveis para aprender e têm menores possibilidades de responder com êxito às exigências da vida escolar. $O$ descuido com pré-escolares parece estar estreitamente ligado a problemas sérios com que se defrontam nossas autoridades de Educação: os altos indices de repetência e evasão escolares nas primeiras séries do sistema de ensino de $1^{\circ}$ grau.

Espósito ${ }^{6}$ refere que, em São Paulo, as taxas de evasão e/ou fracasso escolar na $1^{a}$ série atingem percentagem alarmante ( $50 \%$ das crianças matriculadas). Em termos nacionais, estimou-se 10 que perto de dois milhões e meio de crianças, matriculadas na $1^{a}$ série do $1^{q}$ grau, não conseguem promoção para a série seguinte, o que representa pelo menos $40 \%$ dos alunos que iniciam a vida escolar.

Ante as evidências apresentadas é inegável a necessidade premente e prioritária de atendimento global à criança na idade pré-escolar, para favorecer seu desenvolvimento bio-psíquico e sócio-emocional.

No Brasil, contamos com várias instituições que assistem a pré-escolares, porém geralmente são dispendiosas e não conseguem propiciar cobertura suficiente. Por isso mesmo, foi idealizado e proposto por Gandra ${ }^{7,8}$ um modelo supletivo e nāo convencional de atenção integral ao pré-escolar: - Centro de Educação e Alimentação do Pré-Escolar (CEAPE), com participação obrigatória da mãe da criança assistida.

Entre os objetivos do CEAPE incluem-se os seguintes: melhorar o estado nutricional de pré-escolares, mediante atividades de educação e suplementação alimentar, e promover o desenvolvimento global e harmônico da criança, através da educação e recreação orientada.

Como o CEAPE não pretende antecipar ou acelerar a escolarização da criança, ou seja, não é programa de pré-escolarização, e sim, é modelo que visa precipuamente a favorecer o desenvolvimento sócio-psicomotor do pré-escolar, empregando para tanto a recreação, foi elaborado e é utilizado no CEAPE o Plano Rotativo de Recreação Orientada ${ }^{3}$. Esse instrumento, simples e prático, oferece aos professores e mães de pré-escolares, sugestão de atividades que podem ser desenvolvidas com a criança, num sistema rotativo. A característica "rotativa" do Plano permite, que no rodizio das atividades, seja favorecido o desenvolvimento sócio-psicomotor em seus múltiplos aspectos.

Ante as atividades desenvolvidas com os pré-escolares no CEAPE, cabia esta indagação: tendo-se propiciado condições favoráveis para o desenvolvimento sócio-psicomotor das crianças que frequientaram o CEAPE, teria esse desenvolvimento melhorado sua capacidade de aprendizagem? Responder a tal indagação, constituiu o objetivo do presente estudo.

\section{METODOLOGIA}

Foram levantadas várias subamostras de pré-escolares que freqüentaram 0 CEAPE nos anos de 1975, 1976, 1977 e 1978, a partir dos registros de matricula existentes em unidades CEAPE localizadas em bairros periféricos dos municipios de Leme, Campinas e Capivarí, no Estado de São Paulo. Estas crianças, que totalizaram 268, constituiram o grupo de pré-escolares beneficiados pelo programa CEAPE (Ceapenses), cujo rendimento escolar na $1^{\text {a }}$ série do ensino de $1^{\circ}$ Grau foi avaliado, respectivamente, nos anos de 1976, 1977, 1978 e 1979, em 9 escolas dos 3 municípios estudados.

Um número igual de pré-escolares (268), que não freqüentaram o CEAPE, foi sorteado aleatoriamente, a partir dos registros das escolas de 10 Grau que participaram deste estudo, quando então freqüentavam também a 1a série do 1o Grau, nos anos de $1976,1977,1978$ e 1979 . Antes do sorteio destes pré-escolares, cuidou-se de verificar 
PONTIERI, M.J. et al, Avaliação do aproveitamento escolar de pré-escolares do Programa Centro de Educação e Alímentação do Pré-Escolar. Rev. Saúde públ., S. Paulo, 15(supl.): 148-58,1981.

se os mesmos residiam nas áreas de influência do CEAPE. Este grupo de crianças constituiu a amostra de pré-escolares "Não-Ceapenses" (grupo controle).

Para avaliar o rendimento escolar na 2 série do $1^{\circ}$ Grau, obteve-se, a partir dos resultados obtidos no levantamento feito para a la série, uma amostra de 100 pré-escolares aprovados, dos quais 50 eram "Ceapenses" e os restantes "Não-Ceapenses", verificando-se os resultados de qualificação por eles obtidos na $2^{\text {a }}$ série, nos anos de 1977, 1978 e 1979.

No sentido de alijar qualquer interferência pessoal na qualificação dos pré-escolares. utilizou-se os dados resultantes da avaliaçãc do rendimento escolar, já realizada nas escolas, segundo o modelo recomendado pela Secretaria de Estado da Educação de São Paulo e adotado pelos estabelecimentos de ensino de 1\% Grau.

Nas escolas de 1o Grau, o rendimento escolar é avaliado mediante 5 conceitos (A, B, C, D e E) obtidos em 3 matérias: Língua Portuguesa, Iniciação à Ciência e Integração Social. A cada conceito corresponde um valor determinado de pontos. Assim: $\mathrm{A}=5 ; \mathrm{B}=4 ; \mathrm{C}=3 ; \mathrm{D}=2$ $\mathrm{e} \mathrm{E}=1$. Os conceitos $\mathrm{A}, \mathrm{B}$ e $\mathrm{C}$ aprovam - aluno para a série seguinte, enquanto $D$ e E obrigam a criança à repetência da série cursada.

Apesar da menor sensibilidade do critério usado pelo sistema de ensino para avaliar o rendimento escolar, considerou-se vantajoso o seu emprego neste estudo, por duas razōes :

a) ser este o critério adotado oficialmente para medir o aproveitamento da criança na escola, e principalmente,

b) afastar a possibilidade de eventual favorecimento a um dos dois grupos de crianças estudadas, porquanto, os resultados sobre o rendimento escolar foram retirados dos fichários da Secretaria de Educação local, após o término do ano letivo, com total desconhecimento dos professores que avaliaram os alunos durante o ano escolar. Este aspecto, de grande importância, neste tipo de comparação, compensa largamente a escolha do processo de avaliação adotado.

No decorrer do ano letivo (180 dias úteis) os conceitos de aproveitamento foram dados bimestralmente, segundo os pontos ganhos em cada matéria, por aluno.

$O$ conceito final foi estabelecido utilizando-se a média dos pontos obtidos em cada matéria nos 4 bimestres, sendo que, segundo a norma adotada pela Secretaria de Educação do Estado, a matéria Lingua Portuguesa $\dot{e}$ a que define a aprovaçăo do aluno.

Dado o conceito final, o aluno ainda tem duas outras oportunidades para alcançar o minimo de pontos exigidos para sua promoção à série seguinte: a Recuperação e o Conselho de Classe.

Embora a amostra levantada para medir a rendimento escolar, inicialmente nos parecesse pequena, a constância das diferenças encontradas entre os 2 grupos de crianças nas 9 escolas dos 3 municípios estudados, animou-nos a efetuar uma análise estatística de maior profundidade.

Desta maneira, para a amostra levantada, foram calculados a média e o desvio padrão dos pontos obtidos por cada pré-escolar

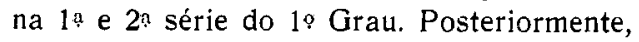
foi aplicado o Teste de Student (test " $t$ "). Os dados obtidos nos encorajaram a apresentar estes resultados preliminares.

\section{RESULTADOS}

Como podemos observar nas Tabelas 1 , 2 e 3, as diferenças encontradas entre os dois grupos de crianças estudadas, respectivamente, nos municipios de Leme, Campinas e Capivari, sugerem que aqueles pré-escolares que frequientaram o Programa CEAPE tiveram um melhor rendimento escolar, o que contribuiu para diminuir o numero de reprovados na 1 a série do 1ọ Grau.

Para o total de pré-escolares $(N=536)$, a Tabela 4 mostra que o Aproveitamento Escolar médio obtido pelas crianças "Ceapenses" e"Não-Ceapenses", na 1 ạ série do 10 Grau, foi, respectivamente, 3,11 e 2,60 pontos. 


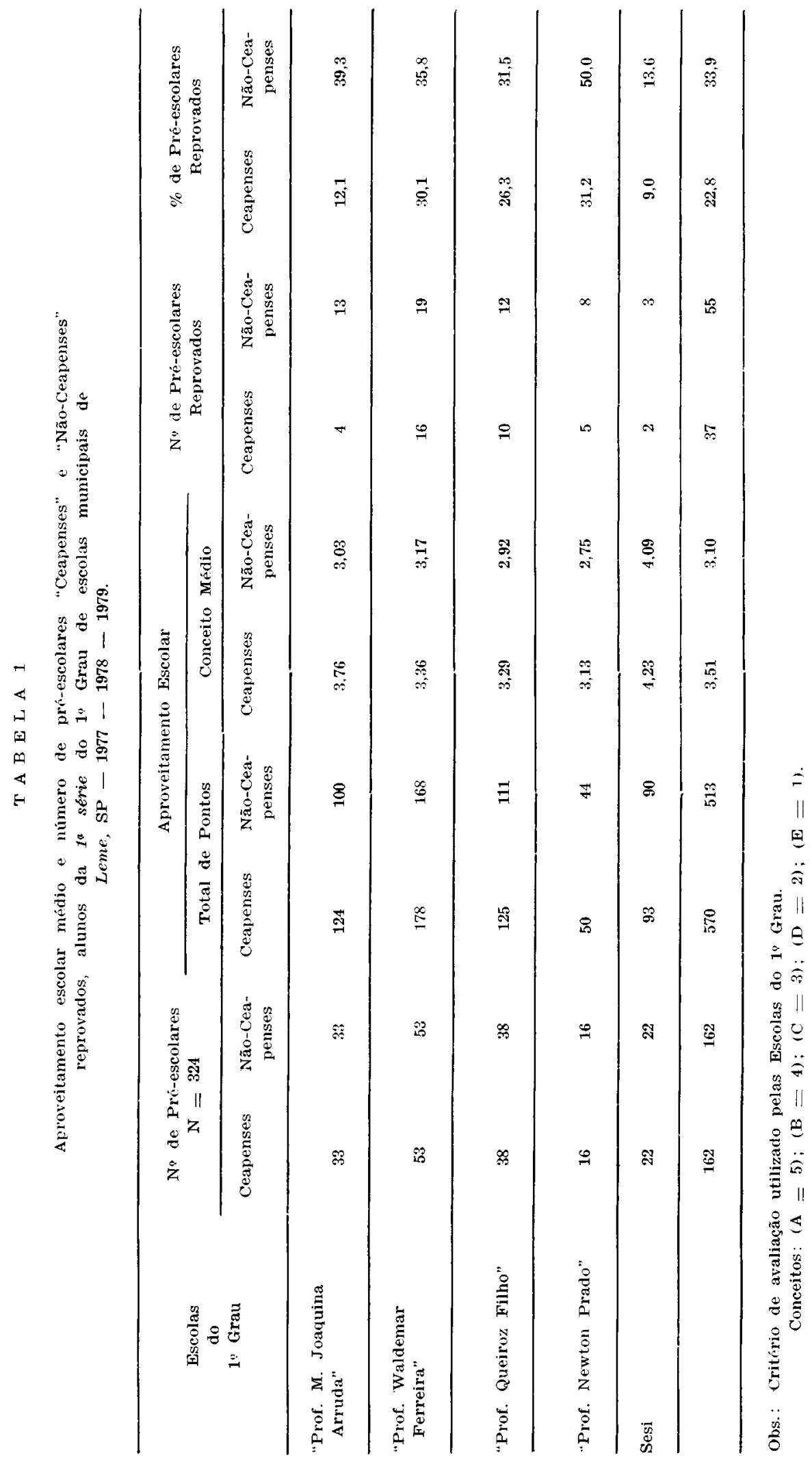




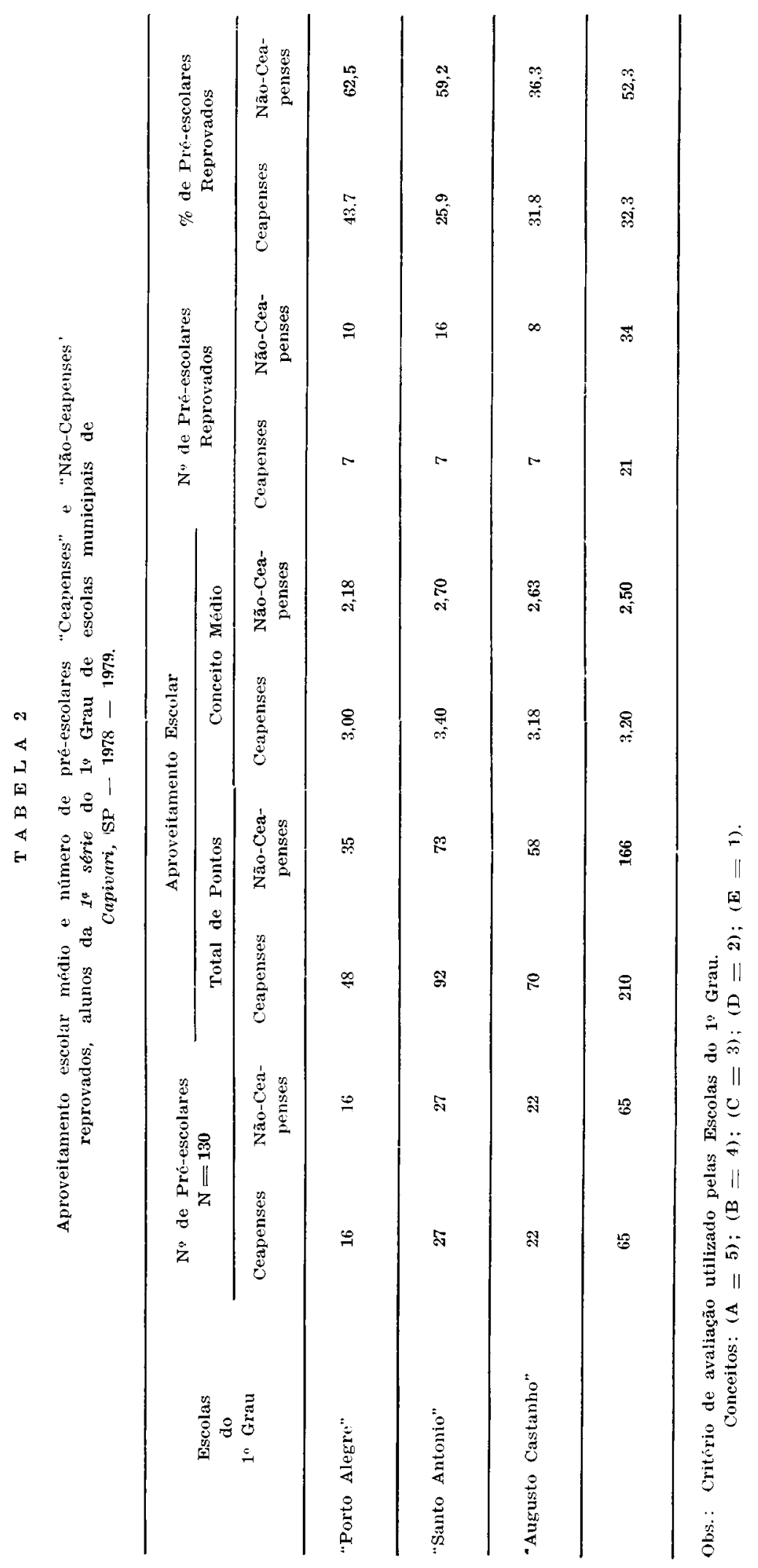




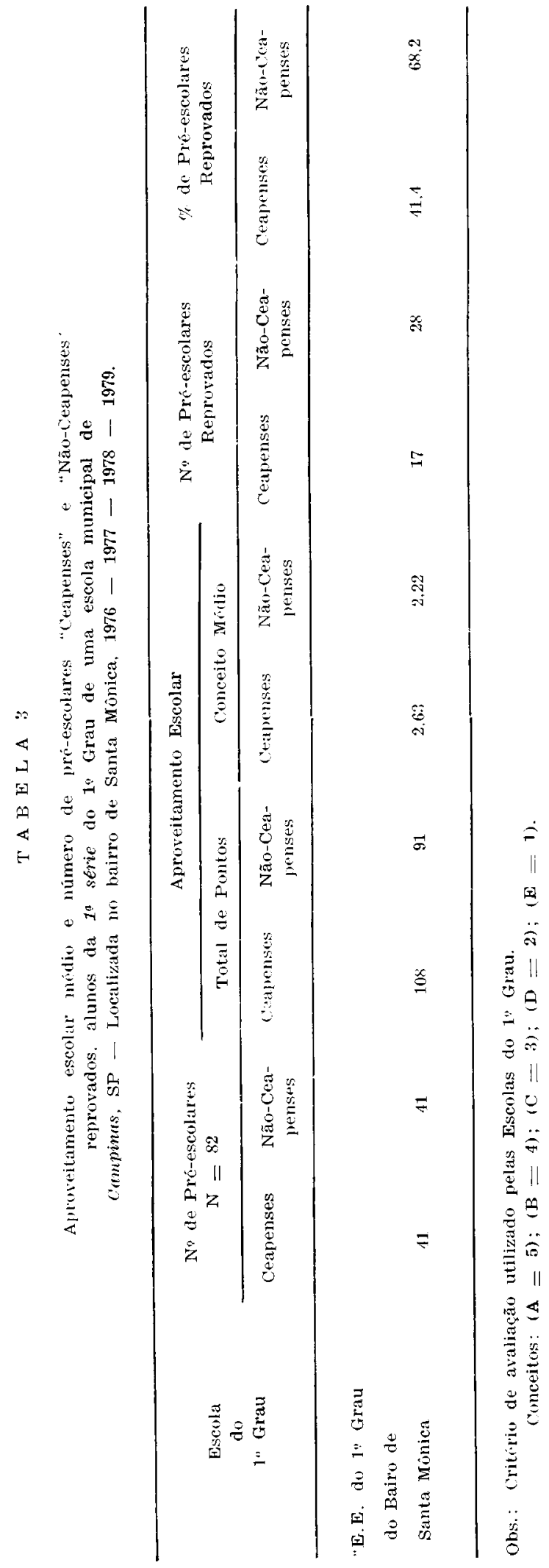




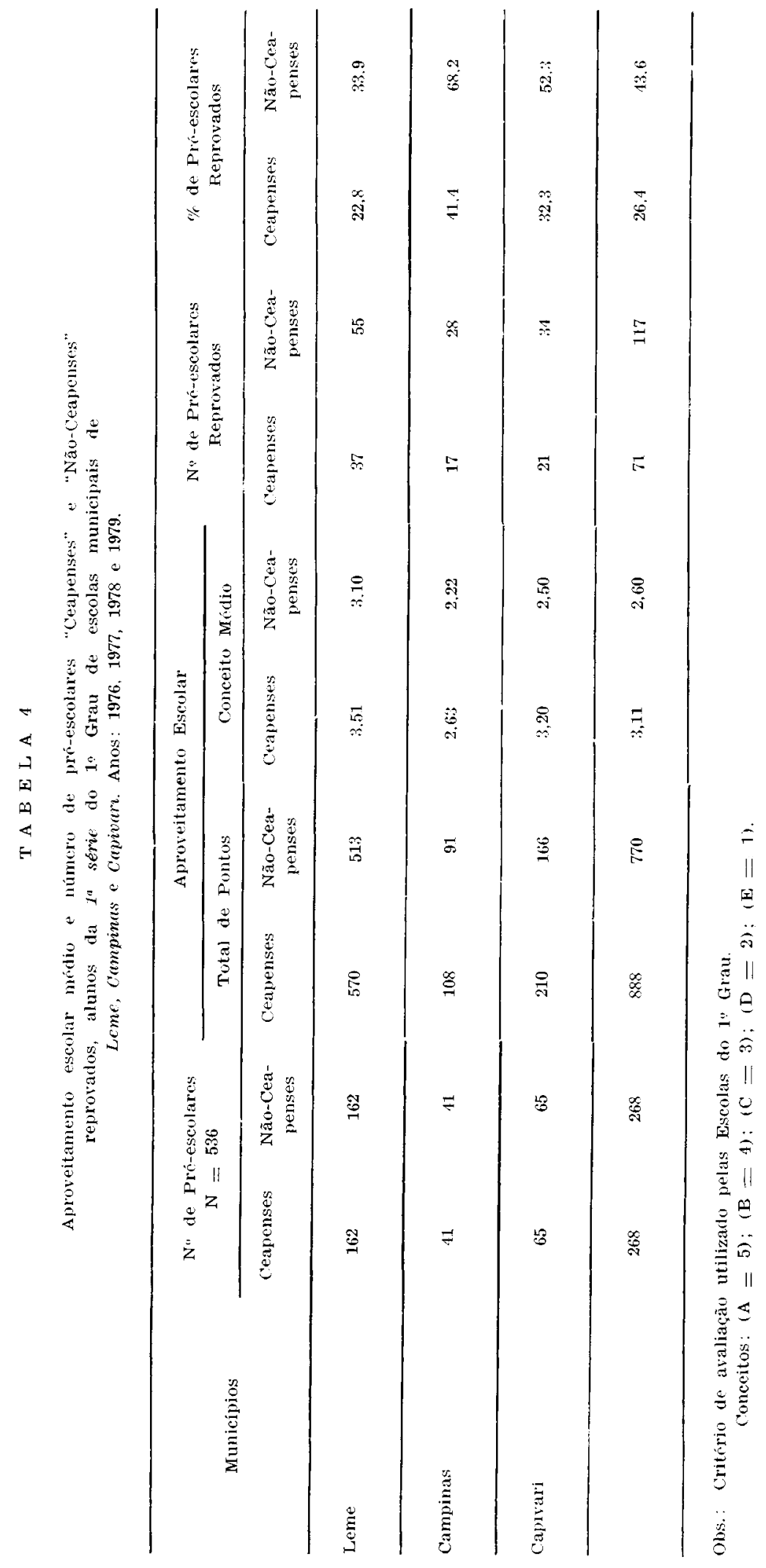


PONTIERI, M.J. et al. Avaliação do aproveitamento escolar de pré-escolares do Programa Centro de Educação e Alimentação do Pré-Escolar. Rev. Saúde públ., S. Paulo, 15(supl.): 148-58,1981.

Se examinarmos o número de pré-escolares reprovados na $1^{\text {a }}$ série do $1^{\circ}$ Grau, podemos verificar que dos 268 pré-escolares "Não-Ceapenses", quase a metade $(43,6 \%)$ foi reprovada, enquanto que $26,4 \%$ das 268 crianças que freqüentaram o CEAPE não obtiveram pontos suficientes para sua promoção.

A Tabela 5 evidencia que para o total de pré-escolares $(\mathrm{N}=100)$, o Aproveitamento Escolar médio obtido pelas crianças "Ceapenses" e "Não-Ceapenses" na $2^{a}$ série do 1? Grau, foi respectivamente, 3,68 e 3,26 pontos.

Quanto ao número de pré-escolares reprovados na $2^{\text {a }}$ série do $1^{\circ}$ Grau, podemos observar na Tabela 5 que dos 50 pré-escolares "Não-Ceapenses", 13 foram reprovados, enquanto que apenas 3 dos 50 pré-escolares que freqüentaram o Programa não conseguiram o número suficiente de pontos para serem promovidos.

Dada a constância das diferenças encontradas nas 9 escolas dos 3 municipios estudados, procedeu-se a análise estatística dos dados, com a finalidade de verificar se os resultados de aproveitamento escolar obtidos pelas crianças, na $1^{\text {th e }} 2^{\text {a }}$ séries do 10 Grau, eram realmente maiores para aquelas crianças que freqüentaram o Programa CEAPE.

Para testar a significância da diferença entre as médias de aproveitamento escolar das duas amostras independentes, o teste estatístico utilizado foi o teste $t$ de Student.

\section{Hipóteses:}

$\mathrm{H}_{0}=$ não há diferenças de aproveitamento escolar para os dois grupos,

$\mathrm{H}_{1}=0$ aproveitamento escolar é maior para o grupo de crianças "Ceapenses".

\section{Estatisticas do test $t$ :}

Regra de Decisão: Rejeito $H_{0}$ se $t_{0}>t_{c}$, onde:

$t_{0}=$ valor da estatistica $t$ observado.

$t_{c}=$ valor tabelado para um nivel de significância $\alpha$ estabelecido.

\section{Resultados do teste $t$ :}

- Ia série do lo Grau

$\mathrm{t}_{\mathrm{o}}=3,8839$

$\mathrm{t}_{c}=\mathrm{t}(\alpha=0,05, \infty$ G.L. $)=1,645$

$t_{o}>t_{c}$, portanto rejeita-se $H_{o}$

- 2a série do 10 Grau

$\mathrm{t}_{\mathrm{o}}=2,7551$

$t_{c_{i}}=\mathrm{t}(\alpha=0,05,60$ G.L. $)=1,671$

$\mathrm{t}_{. .}=\mathrm{t}(\alpha=0,05,120$ G.L. $)=1,658$

$t_{c_{1}}<t_{e}(80$ G.L. $)<t_{c_{2}}$

$t_{0}>t_{c}$, portanto rejeita-se $H_{0}$

Diante dos resultados acima apresentados, fica demontrado que a média de pontos obtidos na $1^{\text {a }}$ e $2^{\text {a }}$ séries do $1^{0}$ Grau foi significantemente maior para aquelas crianças que freqüentaram o Programa CEAPE.

\section{COMENTARIOS E CONCLUSOES}

O exame dos resultados preliminares obtidos na avaliação do aproveitamento escolar de crianças "Ceapenses" e "Não-Ceapenses" leva a alguns comentários pertinentes e a apresentar as conclusões que este estudo sugere.

No CEAPE, ao invés de se deixar ao arbitrio dos professores a seleção de atividades lúdicas para a estimulação de pré-escolares empregou-se um Plano de Recreação Orientada 3, além dos momentos destinados à recreação livre. Essa estratégia permitiu que fossem trabalhadas as diferentes áreas do desenvolvimento sócio-psicomotor dos pré-escolares, o que possivelmente explica a constância dos resultados encontrados em todas as escolas de todas as cidades que participaram deste estudo. Assim, verificou-se que houve diferença no rendimento escolar de crianças "Ceapenses" e "Não-Ceapenses, diferenças estas sempre constantes e significantes. 


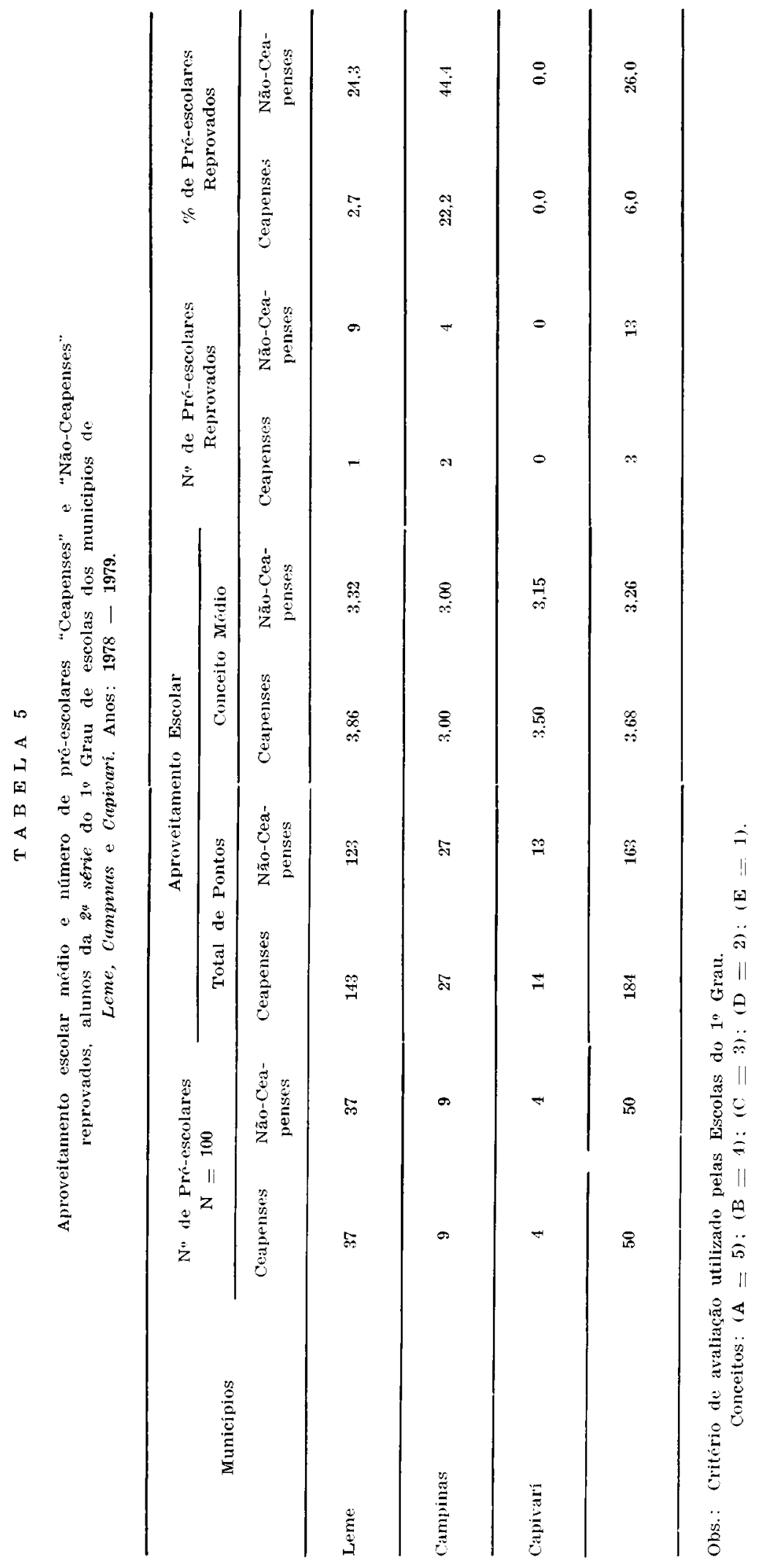


O aproveitamento escolar de pré-escolares "Ceapenses" na 1 a série do 1 \% Grau, avaliado através do sistema da própria Secretaria de Educação do Estado, foi maior do que o de crianças "Não-Ceapenses" em todas as escolas, e continuou sendo maior ao término da $2^{\text {a }}$ série.

O melhor rendimento na escola, de pré-escolares "Ceapenses", coincidiu com a diminuição da repetência na $1^{\text {la }}$ série do 1 o Grau, fato que também se verificou na $2^{\text {a }}$ série, quando a grande maioria das crianças "Ceapenses" conseguiu vencer esta etapa da vida escolar.

Os resultados preliminares obtidos na avaliação do rendimento escolar de pré-escolares "Ceapenses" e "Não-Ceapenses", parecem sugerir ter sido positiva a influência do CEAPE no que concerne ao desenvolvimento integral das crianças que freqüentaram o programa, dotando-as de habilidades que possivelmente contribuiram para que alcançassem maior êxito na escola.

É evidente que este estudo não permite que se tenha a pretensão de extrapolar seus resultados, visto que a amostra examinada não é representativa nem da população pré-escolar, nem dos estabelecimentos públicos de ensino. Todavia, os resultados obtidos indicam que houve diferença significante no aproveitamento escolar entre os dois grupos de crianças estudados; e mais, essa diferença foi constante em todas as escolas estudadas, tanto na $1^{\text {a }}$ quanto na $2^{\text {a }}$ série do 10 Grau. Parece, então, que as diferenças encontradas apontam a direção a seguir: ampliar rapidamente $o$ atendimento ao préescolar, propiciando-lhe atividades que promovam o seu desenvolvimento harmônico e global, favorecendo, assim, o desempenho da criança na escola.

Em trabalhos anteriores já foi demonstrado através da psicometria, o progresso das crianças "Ceapenses" em relação ao grupo controle, nas diferentes áreas do desenvolvimento global do pré-escolar. Este estudo sobre a avaliação do rendimento escolar sugere que mesmo um atendimento menos sofisticado, como o propiciado pelo CEAPE, parece ter influido também na escolarização da criança, tanto em termos do nível de aproveitamento, quanto do rendimento escolar como um todo. Dessa maneira, e considerando que a validade do modelo CEAPE vem sendo comprovada no País 1 seria perfeitamente justificável adotar-se esse tipo de atendimento a pré-escolares no sistema de ensino brasileiro, uma vez que é econômico, eficiente, eficaz e, sobretudo, exeqüível.

FONTIERI, M. J. et al, [Assessment of school performance of preschool children in CEAPE program]. Rev. Saúde públ., S. Paulo, 15(suppl.):148-58, 1981.

ABSTRACT: The scholastic performance of subjects who attended the CEAPE program as compared to a control group belonging the same community which did not attend the program, is assessed. It was demonstrated that performance in 1 st and 2nd grades was significantly better for those students involved in the program. Therefore besides differences in socio-psycho-motor development demonstrated in former studies, the CEAPE, although simple and inexpensive, also garantees better scholastic performance to those attending the program.

UNITERMS: Preschool, child, evaluation. Child development. Learning. CEAPE 
PONTIERI, M.J. et al. Avaliação do aproveitamento escolar de pré-escolares do Programa Centro de Educação e Alimentação do Pré-Escolar. Rev. Saúde públ., S. Paulo, 15(supl.): 148-58,1981.

\section{REFERENCIAS BIBLIOGRAFICAS}

1. ARRUDA, B.K.G. A criança carente. Alim. Nutr., 1:12-4, 1980.

2. CABAK, V. \& NAJ DAVIC, R. Effect on physical and mental development. Arch. Dis. Child., 40:532-4-1965.

3. CAVALCANTI, M.L.F.; GIACOMO, D.: OLIVEIRA, A.M. \& GANDRA, Y.R. Plano rotativo de recreação orientada. Bol. Ofic. OMEP, Rio de Janeiro, 3:5-10, 1976.

4. CHAMPAKAM, C.; SRIKANTIA, S.G. \& GOPALAN, C. Kwashiorkor and mental development. Amer. J. clin. Nutr., $21: 844-52,1968$.

5. CHASE, H.P. \& MARTIN, H.P. Undernutrition and child development. New Engl. J, Med., 282:933-9, 1970.

6. ESPOSITO, Y.L. Desnutrição e cognição. Cad. Pes. Fund. Carlos Chagas, 14:87-96, 1975.

7. GANDRA, Y.R. Asistencia alimentaria por medio de centros de educacion y alimentación del pre-escolar. Bol. Ofic. sanit. panamer., 74:302-14, 1973.
8. GANDRA, Y.R. Nutrition and dietetic education program for preschool children at primary schools in Brazil. In: Anderson, M.A. \& Grewal, T., ed. Nutrition planning in te developing world; proceedings of Regional Workshops held by $C A R E$ ind India, Kenia and Colombia, 1976. Bogotá, Programas Editorales, 1976. p. 205-12,

9. POPPOVIC, A.M.; ESPOSITO, Y.L. \& MALTA CAMPOS, M.M. Marginalização cultural: subsídios para um currículo escolar. Cad. Pesq. Fund. Carlos Chagas, 14:7-23, 1975 .

10. PREFEITURA DO MUNICIPIO DE SAO PAULO, Secretaria de Equcação. $P L A$ NEDI - Plano de Educacão Infantil: doc. bdisico S.M.-5. São Paulo, 1976.

11. SCRIMSHAW. N.S. La malnutrición, el aprendizage y la conducta, Bol. Ofic. sanit. panamer, 65:197-207, 1968.

12. WINICK, M. Malnutrition and brain development. J. Pediat., 74:667-79, 1969.

Recebido para publicasāo em 10/07/1981 Aprovado yara publicacão em 17/11/1981 